\title{
EJPPS
}

EUROPEANJOURNAL OF

PARENTERAL AND

PHARMACEUTICAL SCIENCES

EJPPS - European Journal of Parenteral and Pharmaceutical Sciences Volume 26 Issue 1 https://www.ejpps.online/post/vol26-1-insight-into-the-new-eu-gmp-annex-1 https://doi.org/10.37521/ejpps.26103

\section{Insight into the New EU GMP Annex 1}

F Panofen, PhD, EMEA

D Pandolfi, Systems, Software \& Services

M Della Pietra, Life Sciences

A Campanella

G Artalli, Micro \& Sterility

Corresponding Author: Briana Krueger Technical Writer

Email: bkrueger@pmeasuring.com

Telephone: 1-720-320-5596 


\section{Insight into the New EU GMP Annex 1}

F Panofen, PhD, EMEA, D Pandolfi, Systems, Software \& Services, M Della Pietra, Life Sciences, A Campanella, G Artalli, Micro \& Sterility

\section{Summary}

The 2020 release of the EU GMP Annex 1 draft includes a new chapter on viable and non-viable environmental and process monitoring. The contamination control strategy and its dependence on trend analysis, process understanding, thorough investigation, and a commitment to progress is now an essential activity referenced multiple times through the document. There is also clear differentiation between qualification and monitoring, and quality risk management concepts are critical to successful implementation of system controls.

Key words: Annex 1, quality by design, contamination control strategy (CCS), quality risk management, data trend analysis, risk assessment

\section{Introduction}

In the description of the guideline's scope and principle, risk assessments are the recommended approach for ensuring each controlled area is optimized validated and qualified. Quality objectives are set and fulfilled through the application of quality assurance systems, and these systems are controlled via scientific evaluation of risks in accordance with quality risk management (QRM) principles. Monitoring devices provide the reliable, actionable data necessary to support scientific evaluation, and are best utilized as part of a network of failsafes to prevent unforeseen events (i.e., interventions, system deterioration) from impacting product quality[1]. The document underscores the opportunity for assumptions to undermine sound risk determination. Referenced throughout the Annex $1 \mathrm{draft}$, all decisions and methodology are justified in a document called the contamination control strategy (CCS), an active document for continuous improvement and application. The CCS is not limited to a particular process but is all-encompassing, linking steps as technical as particulate and microbial monitoring to a wider picture of the entire facility and its critical control points.

Figure 1. Critical point of a filling line

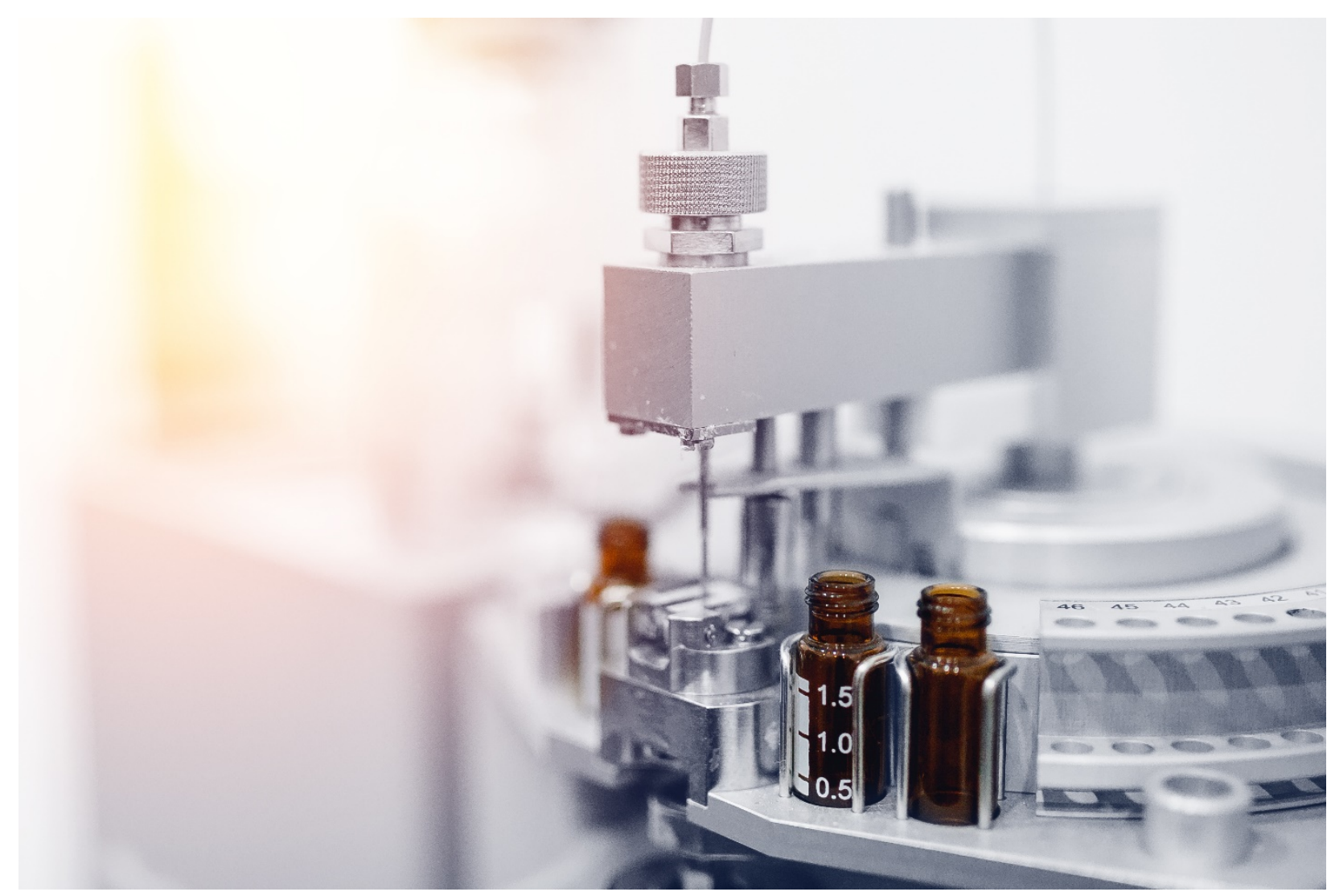

\section{Differentiating Terms}

There are three core events for environmental contamination control in a clean area. In order to understand when and where they occur, they must be differentiated based on purpose. The first and most obvious is monitoring, where the potential contamination risk to product is assessed. It can include additional testing outside of manufacturing areas and operations to identify areas where contamination could be allowed into clean areas. But how are clean areas designated as such? This is the role of qualification, where a classified room's compliance with its intended use is determined. Qualification must be performed every six months for Grade A and Grade B, and every twelve months for Grade C and Grade D. Classification is where the intended use or "cleanliness" of a room is determined, based on ISO 14644-1:2015 regulatory specifications ${ }^{2}$. Classification is a step within qualification and employs the use of monitoring equipment to determine relevant total particle counts for comparison. Refer to EU GMP Annex 15 for the methodology ${ }^{3}$, and ISO 14644-3:2019 for the steps describing the test procedure ${ }^{4}$.

\section{Monitoring and System Design}

QRM systems not only include the facility, equipment and personnel but also the design and layout of their interaction. According to Section 9 of the latest Annex 1 draft, monitoring should be performed to the extent that it can capture any events that occur during the manufacturing process, while also allowing for a timely response by operators. Grade A in particular requires continuous supervision for particulates greater than or equal to 0.5 micrometers, and less than or equal to 5 micrometers (see Table 6 and 7 of the Annex 1 draft for non-viable and viable limits, respectively) with a suitable sample flowrate of at least 28 liters per minute. Once again, the sampling methodology, placement, and orientation are determined by risk assessment. Improved technologies, such as those requiring less operator intervention and training, permit monitoring to remain a non-interference to the product and process. Specific caution is given to the airflow patterns of Grade A and Grade B areas, where the chosen approach to monitoring could make an impact. Due to the wide variety of sampling options, the experts at Particle Measuring Systems offer advisory services, remote or in-person, that can greatly reduce the time this step takes. 


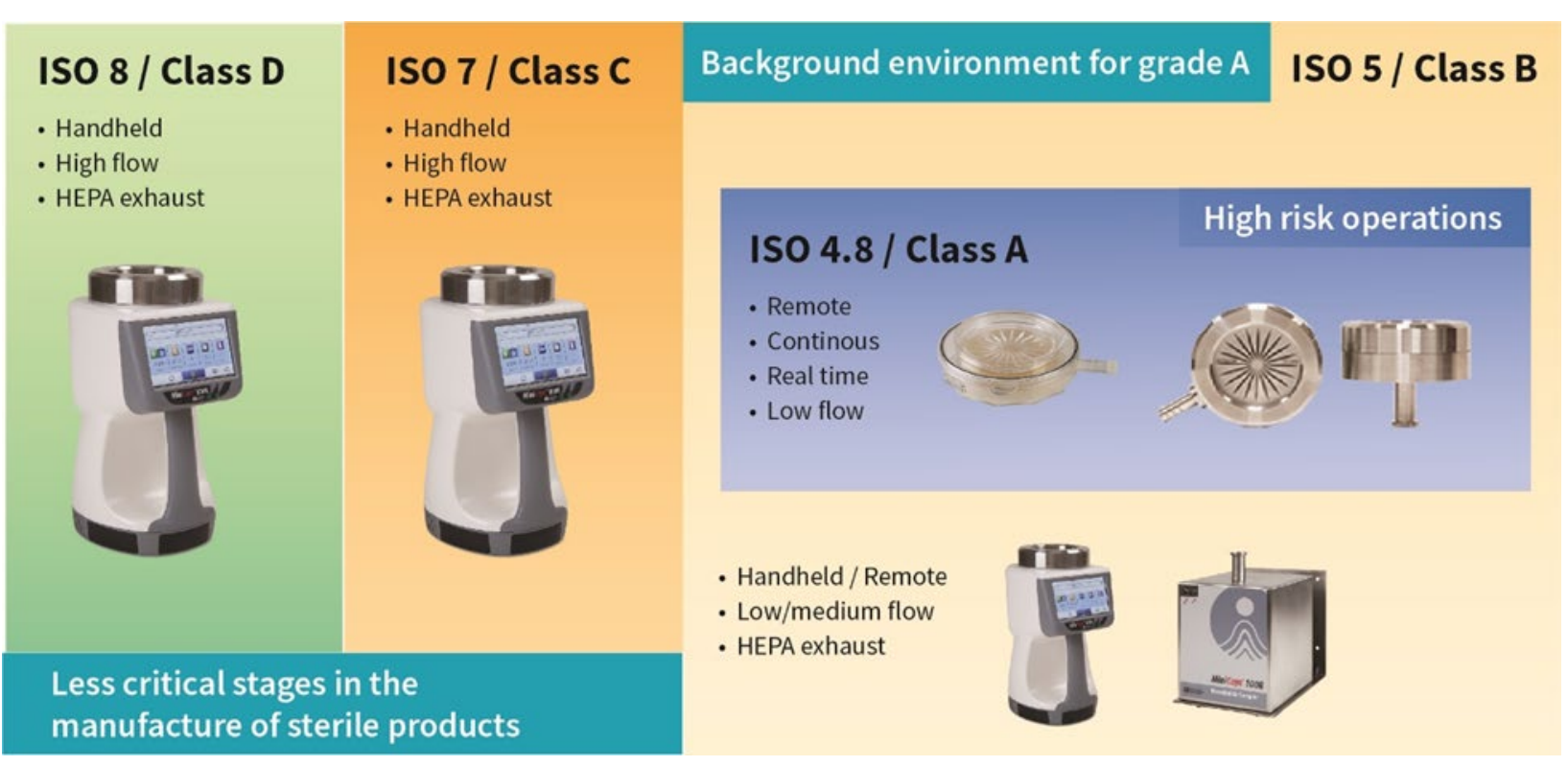

Before choosing which monitoring equipment to use, consider that the method must not only avoid impacting a clean area, but must also be validated to meet or exceed expectations. This is especially important for alternatives to traditional methods (see EP 5.1.6 for more details on validating alternative methods ${ }^{5}$ ). Validated rapid or automated monitoring systems are encouraged by the Annex 1 draft to expedite detection of contamination. Frequent

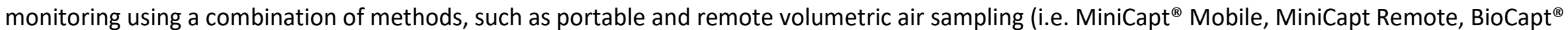
Single-Use, and BioCapt Stainless Steel), swabs, and contact plates is encouraged for viable contaminants. Likewise, traditional methods such as volumetric air devices are recommended for areas with continuous sampling. Relying on snapshots of the environment, such as data collection exclusively performed using 100 LPM devices, are discouraged.

\section{Settle Plates and Single Use}

Settle plates measure the surface contamination rate and not the quality of the air. The results are impacted by air speed, airflow pattern, particle adhesion to surfaces, and particle size. Settle plates do not provide meaningful monitoring data and can be replaced and in some cases made obsolete by single use systems ${ }^{6}$.

The Annex 1 draft describes single use systems (SUS) as made up of bags, filters, tubing, connectors, storage-ware, and sensors. They are primarily used as alternatives or replacements to reusable equipment in the manufacture of sterile medicinal products. Section 8 describes the risks associated with SUS as the following:

- Outer packaging breach (i.e., sterility compromise)

- Pin-hole and leakage

- Contact interaction (e.g., adsorption, leachable formation)

- Particulate contamination

- Integrity testing

- Fragility

- Assembly design

- Increase in number and complexity of operations

- Sterilization (supplier-controlled)

Assessment of SUS suppliers as part of a risk analysis is critical for validation and qualification of the CCS. Particle Measuring Systems offers the BioCapt ${ }^{\circledR}$ Single-Use Microbial Impactor, which mitigates the above described risks with the benefit of being optimized for use in critical areas and continuous sampling while reducing the cost of quality with a strong return on investment. These SUS devices are seamlessly compatible with modern manufacturing requirements in addition to neutralizing VHP and antibiotics.

Figure 3. Settle plate replacement: BioCapt ${ }^{\circledR}$ Single-Use

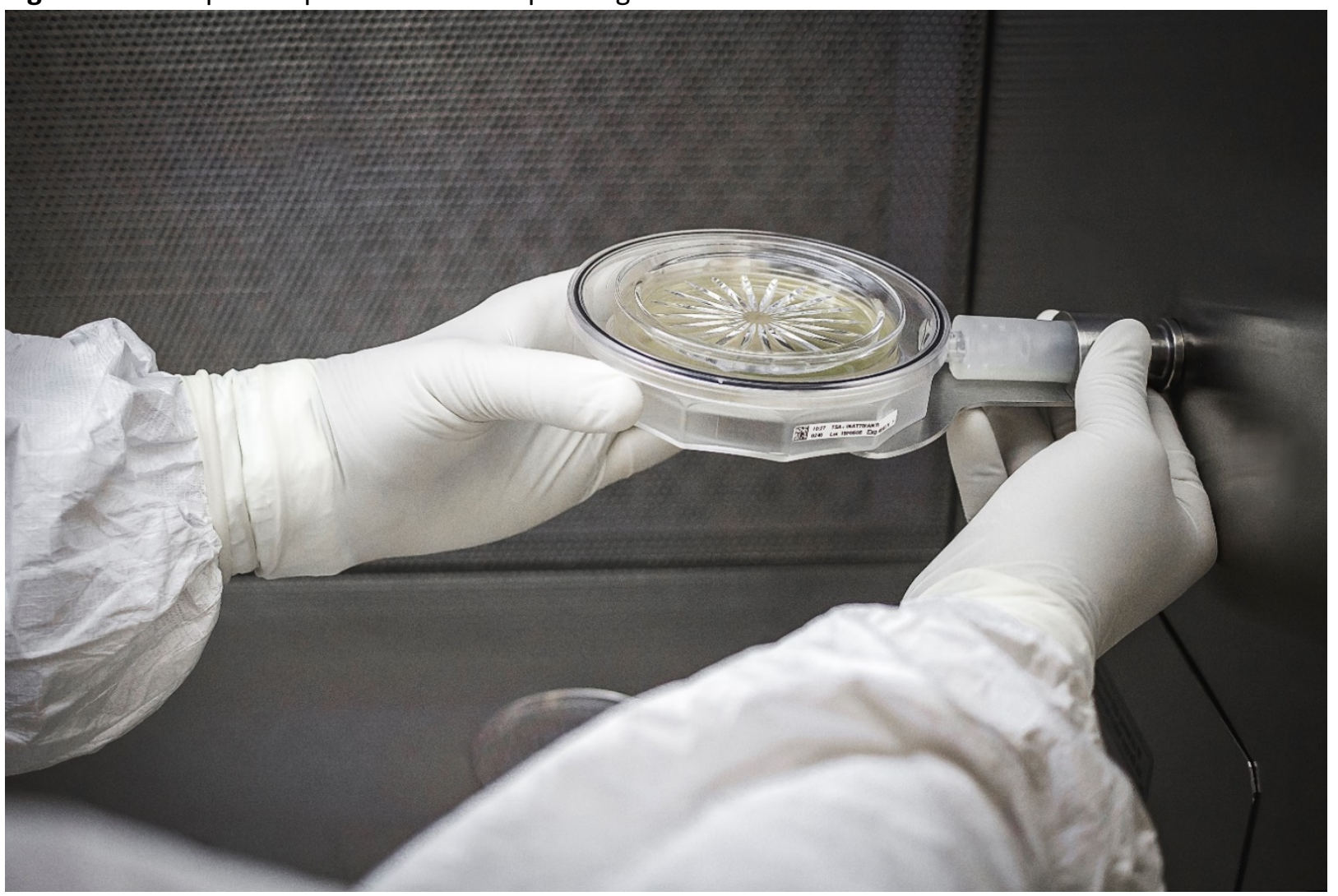


The purpose of microbial testing and monitoring systems is to protect product quality and detect potential contaminants in the environment and product as quickly as possible. To this end, the surrounding environment of Grade A (often designated Grade B) should be monitored with the same approach as Grade A. A comprehensive environmental monitoring program for all areas includes sampling locations, monitoring frequency, monitoring method, and incubation conditions. In essence, these key aspects of the program are the result of many controls occurring simultaneously in the facility. Merely monitoring and testing for its own sake does not ensure sterility.

\section{Cleaning and Disinfection}

The Annex 1 glossary has expanded to include the definitions of cleaning, decontamination and disinfection. The disinfectant's quality-critical parameters or QCPs, such as efficacy or contact time, should be carefully evaluated before determining their appropriate use in the CCS. Keep in mind that alcohol is not always the preferred disinfectant. There are many types of agents adjusted and optimized to suit their application.

Where decontamination efforts are concerned, the draft pays particular attention to materials and equipment that move from a low grade to a high grade, as they pose the highest risk to the final product. Effective disinfection of packaging entering Grade $A$ and Grade B areas should be demonstrated and supported by the CCS, with RABS and isolators cleaned prior to disinfection. Programs for effective cleaning and disinfection procedures, which also includes residue removal, should be written and well-trained to applicable personnel. A successful disinfection process is validated via the study of its effectiveness where used and for the time period before biocide expiration.

Figure 4. Part of cleaning: Disinfectant residue removal

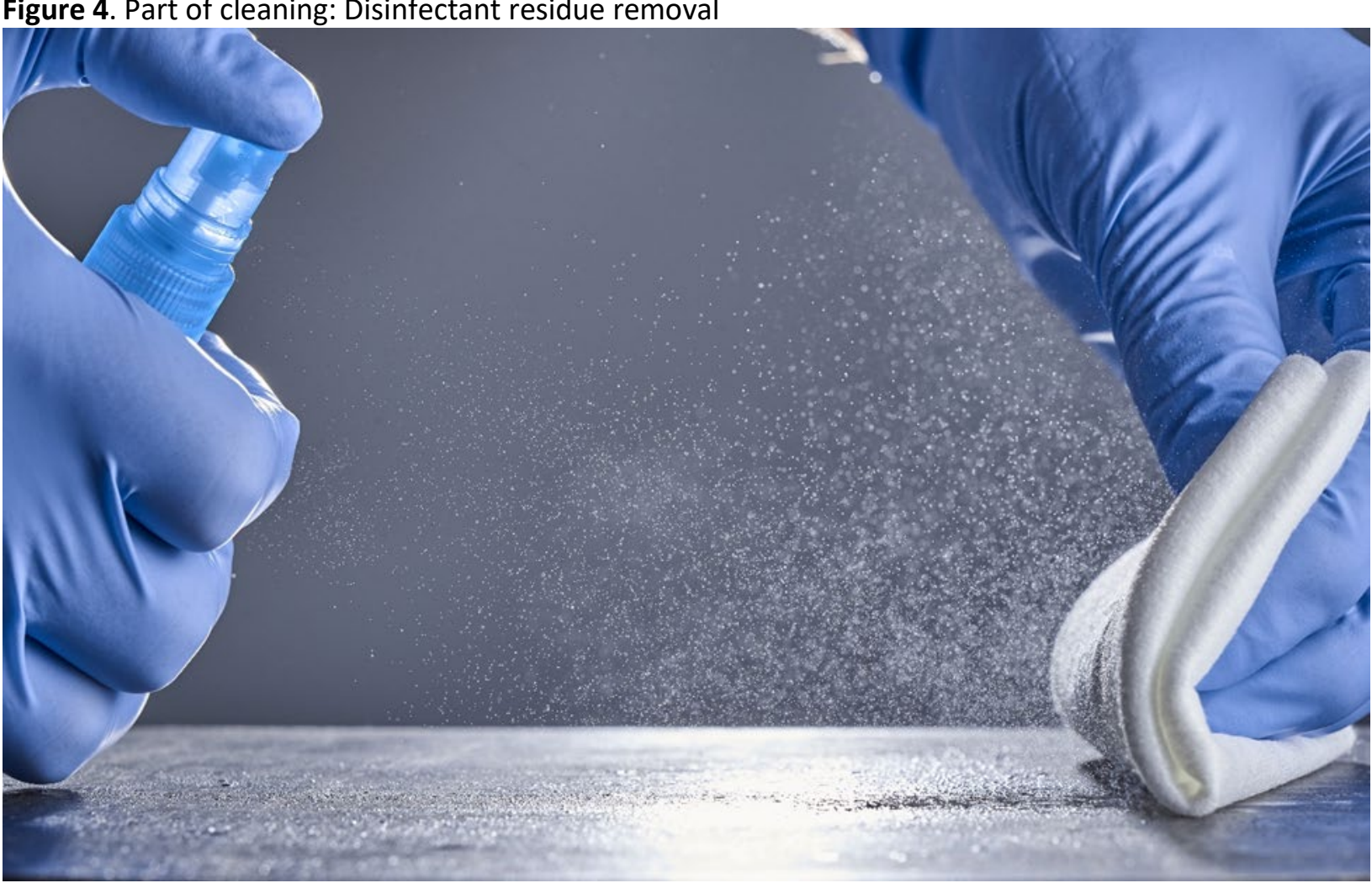

\section{Risk Assessment and Microflora}

Processes, equipment, facilities, and manufacturing activities should be managed in accordance with QRM principles. Risk assessments, which can be performed by third party experts such as Particle Measuring Systems, are conducted based on detailed knowledge of the following:

- Process inputs and outputs

- Facility, equipment, processes, operations, and how they all interconnect

- Historical monitoring data and qualification data

- Typical microbial flora that can be isolated from the environment

Proper incubation conditions are necessary to identify all potential microorganisms in the environment, and if they are not known beforehand, are found during the risk assessment. All data collected is reviewed regularly to verify effectiveness, but the recovery of microorganisms must be shown to verify the methods used for monitoring, disinfection and such are effective. Note that different environmental conditions favor different types of organisms (e.g., anaerobic, slow-growing, and yeasts/molds). Grade A and Grade B do not favor microbiological detection, adding to the challenge.

\section{Events and Deviations}

Alert levels are determined for the quick detection of adverse events before they reach alarm status (sometimes referred to as "action" limits throughout Annex 1). In the short-term, as in the manufacture of one batch of product, total particle counts are kept center-stage to allow for the fastest response to contamination events during active operations. In the long term, viable counts are also reviewed to improve processes and the CCS. Below is the typical flow to setting alert and alarm levels or limits for contamination monitoring: 


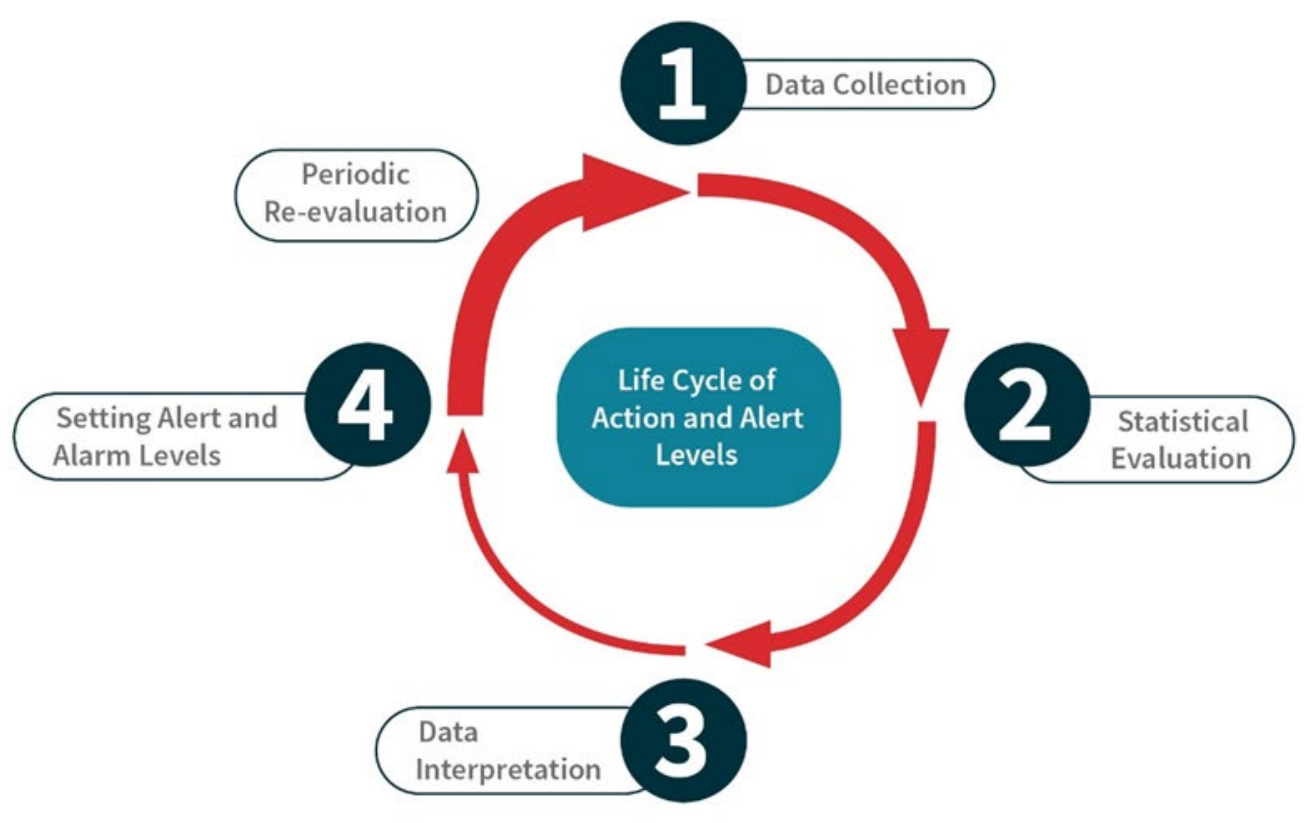

Alert and alarm levels should be based on historical monitoring data. Qualification data may be used in lieu of monitoring data, but the levels should always be open for adjustment based on trending analysis. Scientific justification is necessary when going outside of specified norms, so documentation of any deviations should be made in the CCS. Where there is no specified norm, also document the rationale. Examples include the following:

- Choice of microbial contamination detection method

- Frequency of gown viable monitoring

- Selection of incubation conditions

- Selection of monitoring locations and orientation of applicable devices

- Selection of monitoring sample volumes

- Unidirectional airflow speed, if not in a range of $0.36-0.54 \mathrm{~m} / \mathrm{s}$

Any non-conformities may impact the process and final product, necessitating their investigation. It is also essential to determine the scope, such as entire batches, in the evaluation. Environmental and trend data is part of batch certification (ATMPs) according to Section 10 of the draft, and should they occur, CAPAs require additional sampling to determine their effectiveness. Taking steps to improve facility optimization, data evaluation, and risk mitigation are an ongoing, time-intensive effort with a huge payoff, and advisory teams such as Particle Measuring Systems can greatly help in making them achievable and straightforward.

\section{Conclusion}

The 2020 EU GMP Annex 1 draft has evolved to include viable, non-viable and process monitoring to a degree not previously seen, and outlines the development of a contamination control strategy as part of a facility's essential documentation. This detailed rationale, in addition to trending and data analysis guidelines, risk assessment, and management techniques, is set to become the backbone of quality assurance. The draft's latest updates tell us that in the world of sterile processes, the constantly moving target of quality should not only align with the global effort to harmonize good manufacturing practices, but bring sterile manufacturing to new heights of safety and technological advancement. 


\section{References}

[1] EU Commission. 2020. EU GMP Annex 1 Revision: Manufacture of Sterile Medicinal Products (Draft).

[2] International Organization for Standardization. 2015. ISO 14644-1:2015, Cleanrooms and Associated Controlled Environments, Part 1: Classification of air cleanliness by particle concentration.

[3] EU Commission. 2015. EU GMP Annex 15: Qualification and Validation.

[4] International Organization for Standardization. 2019. ISO 14644-3:2019, Cleanrooms and Associated Controlled Environments, Part 3: Test Methods.

[5] European Pharmacopoeia. 2017. Chapter 5.1.6: Alternative Methods for Control of Microbiological Quality

[6] Dalmaso, Campanella \& Lazzeri. 2020. Continuous and Effective Microbiological Air Monitoring in Critical Environments: A Comparison of Analytical Methodologies. PDA Journal of Pharmaceutical Science and Technology July 2020, 74 (4) 446-455. 